\title{
ON BANACH SPACES WITH MAZUR'S PROPERTY
}

\author{
by DENNY H. LEUNG
}

(Received 10 July, 1989)

1. Introduction. A Banach space $E$ is said to have Mazur's property if every weak* sequentially continuous functional in $E^{\prime \prime}$ is weak* continuous, i.e. belongs to $E$. Such spaces were investigated in [5] and [9] where they were called $d$-complete and $\mu B$-spaces respectively. The class of Banach spaces with Mazur's property includes the WCG spaces and, more generally, the Banach spaces with weak* angelic dual balls [4, p. 564]. Also, it is easy to see that Mazur's property is inherited by closed subspaces. The main goal of this paper is to present generalizations of some results of [5] concerning the stability of Mazur's property with respect to forming some tensor products of Banach spaces. In particular, we show in Sections 2 and 3 that the spaces $E \bar{\otimes}_{\varepsilon} F$ and $L^{p}(\mu, E)$ inherit Mazur's property from $E$ and $F$ under some conditions. In Section 4 , we will also show the stability of Mazur's property under the formation of Schauder decompositions and some unconditional sums of Banach spaces.

We use standard Banach space terminology as found, e.g., in [6]. Throughout, we consider real Banach spaces $E, F, \ldots$, with duals $E^{\prime}, F^{\prime}, \ldots$ The closed unit ball of $E$ is denoted by $U_{E}$. The weak* topology on $E^{\prime}$ is the topology $\sigma\left(E^{\prime}, E\right)$. The $\varepsilon$-tensor product of the spaces $E$ and $F$ is denoted by $E \otimes_{\varepsilon} F$. For a measure space $(\Omega, \Sigma, \mu)$ and $1 \leq p \leq \infty, L^{p}(\Omega, \Sigma, \mu ; E)$ denotes the space of Bochner $p$-integrable (strongly measurable and essentially bounded if $p=\infty) E$-valued functions. We will also write $L^{p}(\mu, E)$ for short, and simply $L^{p}(\mu)$ if $E=\mathbb{R}$. Finally, the cardinality of a set $\Gamma$ is denoted by card $(\Gamma)$.

2. Mazur's property and the $\varepsilon$-tensor product. In [5, Proposition 5.1], it is shown that $E \otimes_{\varepsilon} F$ inherits Mazur's property from $E$ and $F$ if we assume (among other conditions) that $U_{E^{\prime}}$ or $U_{F^{\prime}}$ is weak* sequentially compact. We generalize this result by considering the following weakening of the condition of having a weak* sequentially compact dual ball. Recall that a subset $A$ of a Banach space $E$ is limited in $E$ if every weak* null sequence in $E^{\prime}$ converges uniformly to 0 on $A$.

Definition 2.1. A Banach space $E$ has the Gelfand-Phillips property $(E \in(\mathrm{GP}))$ if every limited set in $E$ is relatively compact.

It is known that $E \in(\mathrm{GP})$ if $U_{E^{\prime}}$ contains a weak* sequentially precompact norming subset, but the converse is false; see, e.g., [8]. For more on the Gelfand-Phillips property, we refer to [3] and [8]. In the following theorem, we consider the space $K_{w} \cdot\left(E^{\prime}, F\right)$ of compact weak ${ }^{*}$-weakly continuous operators from $E^{\prime}$ into $F$. Of course $E \otimes_{\varepsilon} F$ embeds into $K_{w} \cdot\left(E^{\prime}, F\right)$ via the canonical injection $x \otimes y \mapsto\left(x^{\prime} \mapsto\left\langle x, x^{\prime}\right\rangle y\right)$. The two spaces coincide if either $E$ or $F$ has the approximation property.

TheOREM 2.2. Let $E$ and $F$ have Mazur's property. Then $K_{w^{*}}\left(E^{\prime}, F\right)$ have Mazur's property provided the following conditions hold:

(a) either $E \in(\mathrm{GP})$ or $F \in(\mathrm{GP})$; and

(b) the algebraic tensor product $E^{\prime} \otimes F^{\prime}$ is weak ${ }^{*}$ sequentially dense in $\left(K_{w^{*}}\left(E^{\prime}, F\right)\right)^{\prime}$.

Proof. Without loss of generality, assume that $F \in(\mathrm{GP})$. (Note that $K_{w^{*}}\left(E^{\prime}, F\right)$ is isometric to $K_{w^{*}}\left(F^{\prime}, E\right)$ by the map $T \mapsto T^{\prime}$.) Let $\Phi \in\left(K_{w^{*}}\left(E^{\prime}, F\right)\right)^{\prime \prime}$ be weak* sequen-

Glasgow Math. J. 33 (1991) 51-54. 
tially continuous. Define $T: E^{\prime} \mapsto F^{\prime \prime}$ by $\left\langle T x^{\prime}, y^{\prime}\right\rangle=\left\langle x^{\prime} \otimes y^{\prime}, \Phi\right\rangle$ for all $x^{\prime} \in E^{\prime}$ and $y^{\prime} \in F^{\prime}$. Since $\Phi$ is weak ${ }^{*}$ sequentially continuous and $E$ and $F$ both have Mazur's property, it is easy to show that $T$ is $F$-valued and weak*-weakly continuous. We claim that $T U_{E^{\prime}}$ is limited in $F$. Let $\left(y_{n}^{\prime}\right)$ be a weak* null sequence in $F^{\prime}$ and let $\left(x_{n}^{\prime}\right) \subset U_{E^{\prime}}$, it suffices to show that $\left\langle T x_{n}^{\prime}, y_{n}^{\prime}\right\rangle \rightarrow 0$. But this is true since $\left(x_{n}^{\prime} \otimes y_{n}^{\prime}\right)$ is a weak ${ }^{*}$ null sequence in $\left(K_{w^{*}}\left(E^{\prime}, F\right)\right)^{\prime}, \Phi$ is weak* sequentially continuous, and by definition $\left\langle T x_{n}^{\prime}, y_{n}^{\prime}\right\rangle=\left\langle x_{n}^{\prime} \otimes y_{n}^{\prime}, \Phi\right\rangle$. Thus $T U_{E^{\prime}}$ is limited in $F$ and hence relatively compact since $F \in(\mathrm{GP})$. Therefore $T \in K_{w^{*}}\left(E^{\prime}, F\right)$. Obviously, $T$ and $\Phi$ agree on $E^{\prime} \otimes F^{\prime}$ and both are weak* $^{*}$ sequentially continuous. We can thus conclude from part (b) of the hypothesis that $T=\Phi$.

3. Mazur's property in Bochner $L^{p}$ spaces. Throughout this section, let $(\Omega, \Sigma, \mu)$ be a probability space. We will investigate the inheritance of Mazur's property from $E$ to $L^{p}(\mu, E)$. It is well known for $q=p /(p-1), L^{q}\left(\mu, E^{\prime}\right)$ embeds canonically into $L^{p}(\mu, E)^{\prime}$. The following result generalizes Theorem 6.1 in [5].

THEOREM 3.1. Let $E$ be a Banach space with Mazur's property and let $1 \leq p<\infty$. If $L^{q}\left(\mu, E^{\prime}\right)$ is weak ${ }^{*}$ sequentially dense in $L^{p}(\mu, E)^{\prime}$, in particular, if $(\Omega, \Sigma, \mu)$ is a separable measure space, then $L^{p}(\mu, E)$ has Mazur's property.

Proof. Let $\Phi \in L^{P}(\mu, E)^{\prime \prime}$ be weak* sequentially continuous. For $x^{\prime} \in E^{\prime}$ and $A \in \Sigma$, define $v(A)$ by $\left\langle x^{\prime}, v(A)\right\rangle=\left\langle x^{\prime} \chi_{A}, \Phi\right\rangle$. Since $\Phi$ is weak* sequentially continuous and $E$ has Mazur's property, it is readily verified that $v$ is a finitely additive measure with values in $E$. By the Orlicz-Pettis theorem one shows that $v$ is $\sigma$-additive. Clearly $v$ is absolutely $\mu$-continuous. The main step in the proof is the production of a Radon-Nikodým derivative of $v$ with respect to $\mu$. By Proposition 2.4 in [1], it suffices to show that for every increasing sequence $\left(\pi_{n}\right)$ of finite $\Sigma$-partitions of $\Omega$ and, for every $T: L^{1}(\mu, E) \rightarrow c_{0}$, the set

$$
K=\left\{h_{n}:=\sum_{A \in \pi_{n}} \frac{v(A)}{\mu(A)} \chi_{A} ; n \in \mathbb{N}\right\}
$$

has a relatively weakly compact image $T(K)$ in $c_{0}$.

Fix $\left(\pi_{n}\right)$ and $T$ as above. By restriction, we may consider $T$ as a map from $L^{p}(\mu, E)$ to $c_{0}$. There exists a weak* null sequence $\left(F_{m}\right)$ in $L^{p}(\mu, E)^{\prime}$ such that $T(h)=\left(\left\langle h, F_{m}\right\rangle\right)_{m}$ for all $h \in L^{p}(\mu, E)$. Recall that we may identify each $F_{m}$ with a vector measure $F_{m}: \Sigma \rightarrow E^{\prime}\left[2\right.$, p. 115]. Let $E_{n}: L^{p}(\mu, E) \rightarrow L^{p}(\mu, E)$ denote the conditional expectation operator with respect to $\pi_{n}\left[2\right.$, p. 123]. Let $\left(n_{m}\right)$ be an increasing sequence. Since $\left(E_{n}\right)$ is strongly convergent on $L^{p}(\mu, E)$, it is clear that $E_{n_{m}}^{\prime} F_{m} \rightarrow 0$ weak* ${ }^{*}$ Hence

$$
\begin{aligned}
\lim _{m}\left\langle h_{n_{m}}, F_{m}\right\rangle & =\lim _{m}\left\langle\sum_{A \in \pi_{n_{m}}} \frac{v(A)}{\mu(A)} \chi_{A}, F_{m}\right\rangle \\
& =\lim _{m} \sum_{A \in \pi_{n_{m}}}\left\langle v(A), F_{m}(A)\right\rangle / \mu(A) \\
& =\lim _{m} \sum_{A \in \pi_{n_{m}}}\left\langle F_{m}(A) \chi_{A}, \Phi\right\rangle / \mu(A) \quad \text { (by definition of } v \text { ) } \\
& =\lim _{m}\left\langle E_{n_{m}}^{\prime} F_{m}, \Phi\right\rangle=0,
\end{aligned}
$$


since $\Phi$ is weak* sequentially continuous. From this it follows that $\left(F_{m}\right)$ converges uniformly on $K$; hence $T(K)$ is even relatively compact. Therefore, $v$ has a RadonNikodým derivative $h$ with respect to $\mu$. It is clear that $h \in L^{P}(\mu, E)$ and $\langle h, f\rangle=\langle f, \Phi\rangle$ for all $f \in L^{q}\left(\mu, E^{\prime}\right)$. Now the last assumption in the theorem assures us that $h=\Phi$.

\section{Schauder decompositions and sums of Banach spaces.}

THEOREM 4.1. If a Banach space $E$ has a Schauder decomposition $E=\sum_{n=1}^{\infty} E_{n}$, where each summand $E_{n}$ has Mazur's property, then $E$ has Mazur's property.

Proof. Let $x^{\prime \prime} \in E^{\prime \prime}$ be weak* sequentially continuous. For all $n, x_{\mid E_{n}^{\prime}}^{\prime \prime}$ is a weak* sequentially continuous functional in $E_{n}^{\prime \prime}$ and hence is equal to some $x(n) \in E_{n}$. It follows easily from the weak* sequential continuity of $x^{\prime \prime}$ that $\sum_{n=1}^{m} x(n) \rightarrow x^{\prime \prime}, \sigma\left(E^{\prime \prime}, E^{\prime}\right)$, as $m \rightarrow \infty$. It remains to show that $\sum_{n=1}^{\infty} x(n)$ converges in $E$. Assume otherwise, then there are $\varepsilon>0$ and sequences $\left(p_{i}\right),\left(q_{i}\right)$ with $p_{i} \leq q_{i}<p_{i+1}$ for all $i$, such that $\left\|\sum_{n=p_{i}}^{q_{i}} x(n)\right\|>\varepsilon$ for all $i$. Hence there is a normalized sequence $\left(x_{i}^{\prime}\right)$ with $x_{i}^{\prime} \in \sum_{n=p_{i}}^{q_{i}} E_{n}^{\prime}$ such that $\left\langle\sum_{n=p_{i}}^{q_{i}} x(n), x_{i}^{\prime}\right\rangle>\varepsilon$
for every $i$. On the other hand,

$$
\left\langle\sum_{n=p_{i}}^{q_{i}} x(n), x_{i}^{\prime}\right\rangle=\left\langle x_{i}^{\prime}, x^{\prime \prime}\right\rangle \rightarrow 0
$$

since $\left(x_{i}^{\prime}\right)$ is weak ${ }^{*}$ null and $x^{\prime \prime}$ is weak* sequentially continuous. This contradiction proves the claim.

The result above may be extended to some unconditional sums. A cardinal number $\mathbf{m}$ is a real-valued measurable cardinal if there is a real-valued measure defined on all subsets of a set of cardinality $m$ for which points have measure 0 [4, p. 560]. The following is a generalization of Theorem 3.1 in [5].

THEOREM 4.2. If $E$ admits an unconditional Schauder decomposition $E=\sum_{\gamma \in \Gamma} E_{\gamma}$, where each $E_{y}$ has Mazur's property and card $(\Gamma)$ is not real-valued measurable, then $E$ has Mazur's property.

Proof. Let $x^{\prime \prime} \in E^{\prime \prime}$ be weak* sequentially continuous. As in the proof of Theorem 4.1, one verifies that $x_{\mid E_{\gamma}^{\prime}}^{\prime \prime} \in E_{\gamma}$ for all $\gamma$. Let $x(\gamma)=x_{\mid E_{\gamma}^{\prime}}^{\prime \prime}$ for all $\gamma \in \Gamma$. We need to show that (a) $\sum x(\gamma)$ converges in $E$, and (b) $x^{\prime \prime}=\sum x(\gamma)$.

(a) The proof of this part is similar to the proof of Theorem 4.1.

(b) Let $x=\sum x(\gamma)$ and consider $y^{\prime \prime}=x^{\prime \prime}-x \in E^{\prime \prime}$. Clearly $y^{\prime \prime}$ is weak* sequentially continuous. Fix $x^{\prime} \in E^{\prime}$, we may write $x^{\prime}=w^{*}-\sum x^{\prime}(\gamma)$, where $x^{\prime}(\gamma) \in E_{\gamma}^{\prime}$ for all $\gamma$. For $A \subset \Gamma$, let $\mu(A)=\left\langle w^{*}-\sum_{\gamma \in A} x^{\prime}(\gamma), y^{\prime \prime}\right\rangle$. We claim that $\mu$ is $\sigma$-additive. Indeed, if $\left(A_{n}\right)$ is a sequence of subsets of $\Gamma$ which decreases to $\phi$, then $w^{*}-\sum_{\gamma \in A_{n}} x^{\prime}(\gamma) \rightarrow 0$ weak*. Thus $\mu\left(A_{n}\right) \rightarrow 0$ due to the weak ${ }^{*}$ sequential continuity of $y^{\prime \prime}$. Clearly, $\mu$ vanishes on each point of $\Gamma$. Therefore, we must have $\mu=0$ by assumption. In particular, $\left\langle x^{\prime}, y^{\prime \prime}\right\rangle=\mu(\Gamma)=0$. Since $x^{\prime}$ is arbitrary, we must have $y^{\prime \prime}=0$. 
Remark. The measurability assumption on $\Gamma$ cannot be dispensed with as $\ell^{1}(\Gamma)$ fails Mazur's property if card $(\Gamma)$ is real-valued measurable [4, Theorem 5.10].

Corollary 4.3. Let $E$ be a Banach lattice with order continuous norm. If $E$ contains a maximal orthogonal system $\left(x_{\gamma}\right)_{\gamma \in \Gamma}$, where $\operatorname{card}(\Gamma)$ is not real-valued measurable, then $E$ has Mazur's property.

Proof. We refer to [7] for the standard terminology and facts concerning Banach lattices. For each $\gamma$, let $E_{\gamma}$ denote the (projection) band generated by $x_{\gamma}$. The space $E_{\gamma}$ is an order continuous Banach lattice with a weak order unit $x_{\gamma}$. Thus $E_{\gamma}$ is WCG. (In fact, the order interval $\left[-\left|x_{\gamma}\right|,\left|x_{\gamma}\right|\right]$ is a weakly compact generating set.) Hence $E_{\gamma}$ has Mazur's property. Using the order continuity of the norm, we see that $E$ is the unconditional direct sum of $\left(E_{\gamma}\right)_{\gamma \in \Gamma}$. The conclusion now follows from Theorem 4.2.

\section{REFERENCES}

1. J. Batt and W. Hiermeyer, On compactness in $L_{p}(\mu, X)$ in the weak topology and in the topology $\sigma\left(L_{p}(\mu, X), L_{q}\left(\mu, X^{\prime}\right)\right.$, Math. $Z .182(1983), 409-423$. 1977).

2. J. Diestel and J. J. Uhl, Jr., Vector measures, Math. Surveys no. 15 (American Math. Soc.,

3. L. Drewnowski, On Banach spaces with the Gelfand-Phillips property, Math. Z. 193 (1986), 405-411.

4. G. Edgar, Measurability in a Banach space, II, Indiana Univ. Math. J. 28 (1979), 559-579.

5. T. Kappeler, Banach spaces with the condition of Mazur, Math. Z. 191 (1986), 623-631. 1971).

6. H. H. Schaefer, Topological vector spaces, Graduate Texts in Mathematics no. 3 (Springer

7. H. H. Schaefer, Banach lattices and positive operators, Die Grundlehren der Mathematischen Wissenschaften no. 215 (Springer, 1974).

8. T. Schlumprecht, Limited sets in Banach spaces (Dissertation, Ludwig-MaximiliansUniversität, 1987).

9. A. Wilansky, Mazur spaces, Internat. J. Math. Sci. 4 (1981), 39-53.

Department of Mathematics

The University of TeXas at Austin

Austin, TX 78712 